\title{
NOTE ON HARMONIC FUNCTIONS
}

\section{HENRY HELSON 1}

THEOREM. Let $u(r, x)$ be harmonic in the unit circle with the representation

$$
u(r, x)=\sum_{n=-\infty}^{\infty} a_{n} r|n| e^{i n x},
$$

where the complex coefficients $a_{n}$ assume only finitely many values. Suppose further that the mean value

$$
\int_{0}^{2 \pi}|u(r, x)| d x
$$

is bounded for $r<1$. Let $v(r, x)$ be the harmonic function conjugate to $u(r, x)$. Then $u+i v$ is a rational function having all its poles on the boundary of the circle.

The statement of the theorem is close to this result of Szegö: if a power series has coefficients assuming only finitely many distinct values, and if the analytic function so defined can be continued to a point outside the unit circle, then the function is rational with its poles on the circle. (Proofs of this and related theorems are given in [1, Chap. VII].) The referee has kindly called my attention to the following improvement of Szegö's theorem [2]: in the hypothesis it is enough to assume that the coefficients are finite in number, as before, and that the analytic function is bounded in a sector of the circle. The method of proof for the present theorem is quite different.

Actually our result follows easily from the special case when all the $a_{n}$ are either zero or one. In this form it can be restated in several ways:

(a) Let $T$ be a projection in $L(0,2 \pi)$, i.e., a continuous idempotent operator on the summable functions, and suppose $T$ commutes with translations. Then there is a bounded complex measure $\mu$ such that

$$
T f=\mu * f \quad(f \in L),
$$

and $\mu$ consists of finitely many point masses, vanishing singular part, and an absolutely continuous part of the form

$$
\sum_{v=1}^{k} \pm e^{i n_{\nu} x} d x .
$$

Received by the editors January 19, 1953.

${ }^{1}$ Jewett Fellow of the Bell Telephone Laboratories. 
(b) Let $\mu$ be a bounded complex measure defined on the circle, with the property that

$$
\mu * \mu=\mu .
$$

Then $\mu$ is of the form just described.

(c) Let $\left\{n_{\nu}\right\}$ be a sequence of integers which cannot be made periodic by adding or dropping a finite number of elements. Then there is a summable function $f$ on the unit circle with Fourier series

$$
\sum_{n=-\infty}^{\infty} b_{n} e^{i n x}
$$

such that the series

$$
\sum_{\nu} b_{n_{\nu}} e^{i n_{\nu} x}
$$

is not the Fourier series of a summable function. (In this form we have an exact replacement in $L$ for the theorem of $M$. Riesz on conjugate harmonic functions in $L^{p}$ with $p>1$.)

We proceed to the main theorem. By Fatou's theorem, there is a function $F(x)$ of bounded variation on $(0,2 \pi)$ for which

$$
u(r, x)=\frac{1}{2 \pi} \int_{0}^{2 \pi} \frac{1-r^{2}}{1-2 r \cos (x-y)+r^{2}} d F(y)
$$

and the Fourier-Stieltjes coefficients of $d F$ are just the $a_{n}$ :

$$
a_{n}=\frac{1}{2 \pi} \int_{0}^{2 \pi} e^{-i n x} d F(x) .
$$

Associated with $F(x)$ is a complex measure $\mu$, defined and bounded on Borel sets of the circle. By $\mu^{n}$ we mean the measure $\mu$ convoluted with itself $n$ times. If $P(z)$ is any polynomial, $P(\mu)$ is defined, and evidently its $n$th Fourier-Stieltjes coefficient is exactly $P\left(a_{n}\right)$. Suppose the $a_{n}$ assume the $k$ different values $d_{1}, \cdots, d_{k}$. Choose $P(z)$ so that

$$
P\left(d_{1}\right)=d_{1}, \quad P\left(d_{j}\right)=0 \quad(j=2, \cdots, k) .
$$

Denoting $P(\mu)$ by $\mu_{1}$ and defining $\mu_{2}, \cdots, \mu_{k}$ similarly, we have

$$
\mu=\mu_{1}+\mu_{2}+\cdots+\mu_{k},
$$

where the coefficients of each $\mu_{j}$ assume only two values. There is of course a corresponding decomposition of $u(r, x)$. If the theorem can be proved for each part, it evidently holds for the sum. Hence we can 
consider the case where $a_{n}$ takes only two values, and there is no loss of generality in assuming these to be zero and one.

If the coefficients of $\mu$ are all zero or one, we have

$$
\mu * \mu=\mu \text {. }
$$

We have to find all the solutions $\mu$ of this equation. Decompose ${ }^{2} \mu$ into a discrete part consisting of a sum of point masses, $\mu_{0}$, and a continuous part $\mu_{1}$ which vanishes on sets containing a single point. The convolution equation gives

$$
\mu_{0}+\mu_{1}=\mu_{0} * \mu_{0}+2 \mu_{0} * \mu_{1}+\mu_{1} * \mu_{1} .
$$

It is easy to verify that the convolution of two discrete measures is discrete, and so the first term on the right is discrete. Moreover the convolution of a continuous measure by any measure is continuous, ${ }^{3}$ showing that the last two terms on the right are continuous. Equating discrete parts,

$$
\mu_{0} * \mu_{0}=\mu_{0} \text {. }
$$

That is, if $\mu$ is an idempotent measure, the same is true of its discrete component.

Let $\mu_{0}$ have the complex mass $\alpha_{m}$ at points $\lambda_{m}$ on the circle. Then its Fourier-Stieltjes coefficients $a_{n}^{\prime}$ are given by the discrete integral

$$
a_{n}{ }^{\prime}=\frac{1}{2 \pi} \sum_{m=-\infty}^{\infty} \alpha_{m} e^{-i \lambda_{m} n},
$$

where the series converges absolutely. Each term in the series is an almost-periodic sequence in the variable $n$, and since the series converges uniformly in $n$, the sequence $\left\{a_{n}^{\prime}\right\}$ is almost-periodic. But all the $a_{n}^{\prime}$ are either zero or one, so the sequence must actually be periodic. This evidently cannot happen unless all the $\alpha_{m}$ vanish except a finite number, and the $\lambda_{m}$ are situated at roots of unity on the circle. Having characterized the discrete part of $\mu$, we pass to the continuous part $\mu_{1}$.

Since

$$
\mu=\mu_{0}+\mu_{1}
$$

and $\mu_{0}$ is itself idempotent, the coefficients of $\mu_{1}$ are all 0,1 , or -1 .

2 Beurling has used the Lebesgue decomposition of a measure in a similar way.

3 Indeed, the convolution of the measures has Fourier-Stieltjes coefficients which are the products of the coefficients of the given measures. The assertion then follows immediately from the observation that the measure is continuous if and only if its coefficients satisfy $\lim _{N-\infty}(1 / 2 N) \sum_{n-N}^{N}\left|a_{n}\right|^{2}=0$. 
We shall prove that all of them vanish except a finite number, and this is the crux of the theorem. In fact we establish the following more general fact.

Lemma. Let $\nu$ be a continuous measure whose Fourier-Stieltjes coefficients $b_{n}$ all satisfy one of the conditions

$$
b_{n}=0 \text { or }\left|b_{n}\right| \geqq \eta>0 .
$$

Then only finitely many $b_{n}$ are different from zero.

Let $\nu_{0}$ be the singular component of $\nu$, and form the non-negative measure $\left|\nu_{0}\right|$ which assigns to a set $E$ the total variation of $\nu_{0}$ on $E$. Then $\left|\nu_{0}\right|$ is a singular measure. We assert that there is a function $f(z)$ analytic and bounded in the unit circle, having radial boundary value equal to zero everywhere except on a null-set of the measure $\left|\nu_{0}\right|$.

To see that, ${ }^{4}$ form the Poisson integral

$$
U(r, x)=\frac{1}{2 \pi} \int_{0}^{2 \pi} \frac{1-r^{2}}{1-2 r \cos (x-y)+r^{2}} d\left|\nu_{0}(y)\right|
$$

defining a non-negative harmonic function in the circle. The argument used in the proof of Fatou's theorem can be modified to show that

$$
\lim _{r=1} U(r, x)=\infty
$$

at every point where the monotonic function

$$
H(x)=\left|\nu_{0}\right|(0, x)
$$

has infinite derivative. This is the case except on a set which has measure zero $\left(\left|\nu_{0}\right|\right)$. Now let $V(r, x)$ be conjugate to $U(r, x)$, and set

$$
f\left(r e^{i x}\right)=e^{-U-i \boldsymbol{V}} \text {. }
$$

Since $U$ is non-negative, $f(z)$ has bound one in the unit circle, and evidently has the required radial limits. It is essential to observe further that $f(0) \neq 0$.

For any measure $\lambda$ on the circle and fixed $r<1$, define a new measure $f_{r} \cdot \lambda$ by setting

$$
f_{r} \cdot \lambda(E)=\int_{E} f\left(r e^{i x}\right) d \lambda(x)
$$

for any Borel set $E$. For the total variation of $f_{r} \cdot \nu_{0}$ we have

- Dr. Wermer suggested the idea of this proof. The existence of such a function is probably known. 


$$
\lim _{r=1} \int_{0}^{2 \pi}\left|f\left(r e^{i x}\right)\right| d\left|\nu_{0}(x)\right|=0
$$

by the Lebesgue convergence theorem, since the integrand converges boundedly to zero almost everywhere for the measure $\left|\nu_{0}\right|$. The conclusion we need is that the coefficients of $f_{r} \cdot \nu_{0}$,

$$
c_{n}(r)=\frac{1}{2 \pi} \int_{0}^{2 \pi} e^{-i n x} f\left(r e^{i x}\right) d \nu_{0}(x),
$$

tend to zero uniformly in $n$ as $r$ approaches 1 .

Let $f(z)$ have the power series expansion

$$
f\left(r e^{i x}\right)=\sum_{n=0}^{\infty} \gamma_{n} r^{n} e^{i n x}
$$

We have written $b_{n}$ for the coefficients of $\nu$; denote by $b_{n}(r)$ those of $f_{r} \cdot \nu$. Computing with series which converge absolutely for $r<1$,

$$
b_{n}(r)=\sum_{m=0}^{\infty} \gamma_{m} r^{m} b_{n-m},
$$

and the same formula holds for $c_{n}(r)$ in terms of the coefficients $c_{n}$ of $\nu_{0}$.

It is known [3] that for a continuous measure $\nu$, the FourierStieltjes coefficients are small in the sense that

$$
\lim _{N=\infty} \frac{1}{N} \sum_{n=-N}^{N}\left|b_{n}\right|^{2}=0
$$

On account of our hypothesis on the size of the $b_{n}$, it follows that the coefficients must present arbitrarily large gaps. That is, assuming that infinitely many $b_{n}$ are actually different from zero, we can find for any integer $N$ an index $p$ such that

$$
b_{p} \neq 0, \quad b_{p-k}=0 \quad(k=1, \cdots, N) .
$$

The convolution above can be written

$$
b_{p}(r)=\gamma_{0} b_{p}+\sum_{m=N+1}^{\infty} \gamma_{m} r^{m} b_{p-m} .
$$

Hence

$$
\left|b_{p}(r)\right| \geqq\left|\gamma_{0}\right| \eta-\sum_{m=N+1}^{\infty}\left|\gamma_{m}\right| r^{m}\left|b_{p-m}\right|
$$


For fixed $r$, letting $N$ and $p(N)$ increase indefinitely, we conclude

$$
\lim \sup \left|b_{n}(r)\right| \geqq\left|\gamma_{0}\right| \eta>0 .
$$

Now the measure $f_{r} \cdot\left(\nu-\nu_{0}\right)$ is absolutely continuous, and its coefficients tend to zero by the Riemann-Lebesgue theorem. Subtracting them from the $b_{n}(r)$ gives

$$
\lim \sup \left|c_{n}(r)\right| \geqq\left|\gamma_{0}\right| \eta \quad(r<1) .
$$

But we know $c_{n}(r)$ tends to zero uniformly as $r$ tends to 1 . This contradiction shows that only finitely many $b_{n}$ are different from zero, proving the lemma.

Now $\mu$, which was an arbitrary idempotent measure, has been characterized as the sum of a finite set of point masses, and a continuous (moreover evidently absolutely continuous) measure whose coefficients are almost all zero. We have denoted the harmonic function obtained by the Poisson integral from $\mu$ by $u(r, x)$, and its conjugate harmonic function by $v(r, x)$. Then $u+i v$ has the representation

$$
\frac{1}{2 \pi} \int_{0}^{2 \pi} \frac{e^{i x}+z}{e^{i x}-z} d \mu(x)+c \quad(|z|<1)
$$

and it is easy to verify that this is a rational function when $\mu$ is of the given type. This completes the proof of the theorem.

\section{REFERENCES}

1. L. Bieberbach, Lehrbuch der Funktionentheorie, vol. 2, Leipzig, 1934.

2. R. J. Duffin and A. C. Schaeffer, Power series with bounded coefficients, Amer. J. Math. vol. 67 (1945) pp. 141-154.

3. S. Bochner, Vorlesungen uber Fouriersche Integrale, New York, 1948.

YaLe UNIVERSITY 